\title{
CPAP Adherence: A Matter of Perfect Airflow Curves?
}

Positive airway pressure (PAP) remains the first-line treatment of moderate to severe obstructive sleep apnea; however, the effectiveness of PAP depends on adherence. ${ }^{1}$ Although regular PAP use is generally defined as $4 \mathrm{~h}$ of use on $70 \%$ of days ${ }^{2}$ and is still the base for reimbursement in different countries, it is not enough in all patients. Some people will feel better with 2-3 h of use; however, most people need more, and there appears to be a linear relationship between hours of use and impact on sleepiness. ${ }^{3}$ Nevertheless, a recent systematic literature review, evaluating different PAP trials, showed a weighted mean nightly CPAP non-use of $36 \%$ of the night, based on a 7-h/night sleep time, whereas $10.7 \%$ of subjects were unable to tolerate PAP and stopped this treatment. ${ }^{4}$

Non-adherence is an issue, especially taking into account that over the last decade, the patient population presenting for PAP treatment has been changing. More and more patients with mild sleepiness or without sleepiness are treated because of the associated cardiovascular risk. Getting non-sleepy patients to adhere to PAP is challenging. Even the most recent randomized controlled trials evaluating the effects of PAP on long-term adverse cardiovascular outcome risk remain disappointing, and, although not proven, poor adherence due to lack of sleepiness appears to be a reason. ${ }^{5,6}$ On the other hand, of Peker et al ${ }^{6}$ also illustrated that PAP treatment is feasible in non-sleepy subjects if they receive enough education to become motivated. PAP treatment of non-sleepy patients with obstructive sleep apnea demands effort, and PAP treatment of patients with psychiatric comorbidity is even a bigger challenge because PAP adherence was shown to be significantly lower in these patient populations. ${ }^{7}$

In this issue of RESPIRATORY CARE, Krakow et al ${ }^{8}$ evaluate the use of a re-titration protocol (REPAP) in PAP failure subjects, of which 70\% reported co-occurring psychiatric disease. This protocol includes technical solutions emphasizing the handling of mask issues and attempts to eliminate all breathing events, including respiratory effort-related arousals, while also addressing expiratory pressure intolerance (to titrate out respiratory effort-related arousals, higher pressure is

The authors have disclosed no conflicts of interest.

Correspondence: Dries Testelmans MD PhD, University Hospitals Leuven, Herestraat49,3000 Leuven, Belgium.E-mail: dries.testelmans@uzleuven.be.

DOI: $10.4187 /$ respcare. 05498 needed, which may induce expiratory pressure intolerance). In previous work, ${ }^{9}$ the same authors demonstrated that failing to attend to respiratory effort-related arousals and expiratory

See the Original Study on Page 396

pressure intolerance adversely influences PAP adherence. Therefore, in the present patient population, they performed meticulous re-titration(s) using auto-adjusting devices, but they manually overrode the system as necessary. Subjects were discharged with these more sophisticated devices and settings, if necessary. To adequately treat them, often multiple re-titrations were necessary with switches to different PAP modes.

The use of this protocol led to re-initiation of PAP treatment in $72 \%$ of the subjects. Without doubt, it is encouraging to learn that several of the subjects with PAP failure could ultimately be efficiently treated with PAP therapy. So, should we apply the REPAP protocol, including multiple in-laboratory re-titrations, for all patients with PAP failure?

Overall, PAP rejection and non-adherence are complex issues with a myriad of potential etiologies. It is well established that PAP rejection and/or non-adherence can be minimalized by educational and behavioral approaches and by supportive interventions for problem-solving or troubleshooting difficulties related to common adverse effects of the PAP mask, pressure, and device..$^{10}$ It is therefore not surprising that a substantial proportion of the subjects in the REPAP protocol, when asked for reasons for the cessation of their former PAP therapy, mentioned mask discomfort, inability to adapt to PAP, and adverse effects from PAP use. However, it is alarming that a large majority of these subjects verbally indicated that these factors were not addressed before the termination of their initial PAP therapy in the original sleep laboratory.

Theoretically, the efficacy of CPAP can be evaluated by performing a repeat full-night attended titration polysomnography in the sleep laboratory or by home testing with thorough review of objective data related to pressure delivery, leaks, and residual respiratory events from the PAP device. The latter home testing procedures appear more cost-beneficial. However, it should be noted that each PAP manufacturer has its own algorithms for the evaluation and definition of respiratory events and, in the case of an auto-adjusting device, its own algorithms for the pressure change after a breath- 


\section{EDITORIALS}

ing event. Because of industry proprietary rights, it is difficult to gain complete insight into these algorithms. The American Thoracic Society stated in their 2013 report that, based on the available literature data at that time, only the ends of the spectrum of residual events (very high or low values) appear clinically meaningful, and the American Thoracic Society board asked for the performance of more validation studies on this topic. ${ }^{2}$ In this respect, a very recent European randomized study demonstrated a good correlation between the number of residual breathing events evaluated by a specific PAP device and the number detected on polysomnography. ${ }^{11}$ However, the number of residual events was low (due to meticulous titration of PAP beforehand), and the selected group of subjects did not present associated sleep disorders, including severe insomnia, or did not use sedatives or other drugs that might impair sleep. The latter problems are often encountered in patients with psychiatric co-morbidities; consequently, we fully agree with the authors of the present article that in case (re-)titration is necessary in such a patient population, it should be performed in an attended sleep laboratory. ${ }^{8}$

The use of the REPAP protocol led to re-initiation of PAP treatment in $72 \%$ of the subjects, with psychiatric comorbidity present in the large majority. This also implies that $28 \%$ of the included subjects did not use PAP in the end, despite the frequent use of multiple re-titrations. Therefore, another, and in our opinion highly important, issue should be taken into account. In patients with, but also without, psychiatric comorbidity and PAP failure, one should dare to question the original obstructive sleep apnea diagnosis. A review of the initial diagnostic polysomnography could be of major importance. In this trial, the mean duration of time between the original diagnostic polysomnography and the initial re-titration was $>5 \mathrm{y}$; consequently, a new diagnostic polysomnography could be indicated to evaluate whether the diagnosis of obstructive sleep apnea is still valuable and to detect interfering factors (eg, central respiratory events and movements). This diagnostic re-evaluation could also guide the choice between alternative therapies, including non-PAP options.

Nevertheless, Krakow et al ${ }^{8}$ showed that a major subset of PAP failure subjects could be adequately treated, after meticulous re-titration to eliminate respiratory effort-related arousals and expiratory pressure intolerance. These intriguing findings confirm data from a previous publication of the same group indicating that failure to attend to respiratory effortrelated arousals and expiratory pressure intolerance adversely influences PAP adherence. ${ }^{9}$ However, the additional benefit to completely wipe out all respiratory events and to "round" the inspiratory and expiratory airflow curve needs to be further explored and demonstrated by other research groups. Moreover, in the future, more tools are needed to select those patients who really need to be switched to another PAP mode and to better guide the initial PAP mode (to minimalize the number of re-titrations necessary). Meanwhile, it is the duty of every physician involved with patients receiving PAP to closely evaluate and follow up their patients and maximally address any adverse effects.

\section{Dries Testelmans MD PhD \\ Bertien Buyse MD PhD}

Department of Pulmonology and Leuven University Centre for Sleep and Wake Disorders (LUCS)

University Hospitals Leuven

Department of Clinical and Experimental Medicine

KU Leuven

Leuven, Belgium

\section{REFERENCES}

1. Sharples LD, Clutterbuck-James AL, Glover MJ, Bennett MS, Chadwick R, Pittman MA, Quinnell TG. Meta-analysis of randomised controlled trials of oral mandibular advancement devices and continuous positive airway pressure for obstructive sleep apnoea-hypopnoea. Sleep Med Rev 2016;27:108-124.

2. Schwab RJ, Badr SM, Epstein LJ, Gay PC, Gozal D, Kohler M, et al. An official American Thoracic Society statement: continuous positive airway pressure adherence tracking systems: the optimal monitoring strategies and outcome measures in adults Am J Respir Crit Care Med 2013;188(5):613-620.

3. Weaver TE, Maislin G, Dinges DF, Bloxham T, George CF, Greenberg $\mathrm{H}$, et al. Relationship between hours of CPAP use and achieving normal levels of sleepiness and daily functioning. Sleep 2007;30(6):711-719.

4. Rotenberg BW, Murariu D, Pang KP. Trends in CPAP adherence over twenty years of data collection: a flattened curve. J Otolaryngol Head Neck Surg 2016;45(1):43-51.

5. McEvoy RD, Antic NA, Heeley E, Luo Y, Ou Q, Zhang X, et al. CPAP for prevention of cardiovascular events in obstructive sleep apnea. N Engl J Med 2016;375(10):919-931.

6. Peker Y, Glantz H, Eulenburg C, Wegscheider K, Herlitz J, Thunström E. Effect of positive airway pressure on cardiovascular outcomes in coronary artery disease patients with nonsleepy obstructive sleep apnea: the RICCADSA randomized controlled trial. Am J Respir Crit Care Med 2016;194(5):613-620.

7. Means MK, Ulmer CS, Edinger JD. Ethnic differences in continuous positive airway pressure (CPAP) adherence in veterans with and without psychiatric disorders. Behav Sleep Med 2010;8(4):260-273.

8. Krakow B, Ulibarri VA, McIver ND, Yonemoto C, Tidler A, Obando J, et al. Reversal of CPAP failure with the REPAP re-titration protocol. Respir Care 2017;62(4):396-408.

9. Krakow B, Ulibarri VA, Romero E, Thomas RJ, McIver N. Adaptive servo-ventilation therapy in a case series of patients with co-morbid insomnia and sleep apnea. J Sleep Disord Treat Care 2013;2(1):1-10. doi: 10.4172/2325-9639.1000107.

10. Weaver TE, Sawyer AM. Adherence to continuous positive airway pressure treatment for obstructive sleep apnoea: implications for future interventions. Indian J Med Res 2010;131:245-258.

11. Gagnadoux F, Pevernagie D, Jennum P, Lon N, Loiodice C, Tamisier $\mathrm{R}$, et al. Validation of the System One RemStar Auto A-Flex for obstructive sleep apnea treatment and detection of residual apneahypopnea index: a European randomized trial. J Clin Sleep Med 2017; pii: jc-00176-16. [Epub ahead of print] 